(2) Open Access Full Text Article

\title{
Downregulation of VEGFA inhibits proliferation, promotes apoptosis, and suppresses migration and invasion of renal clear cell carcinoma
}

This article was published in the following Dove Press journal:

OncoTargets and Therapy

I2 April 2016

Number of times this article has been viewed

\author{
Fan-Chang Zeng ${ }^{1,2}$ \\ Ming-Qiang Zeng' \\ Liang Huang' \\ Yong-Lin $\mathrm{Li}^{\prime}$ \\ Ben-Min Gao' \\ Jun-Jie Chen' \\ Rui-Zhi Xue' \\ Zheng-Yan Tang' \\ 'Department of Urology, Xiangya \\ Hospital, Central South University, \\ Changsha, ${ }^{2}$ Department of Urology, \\ Hainan General Hospital, Haikou, \\ People's Republic of China
}

Objective: The aim of this study was to investigate the effects of vascular endothelial growth factor A (VEGFA) on cell proliferation, apoptosis, migration, and invasion in renal clear cell carcinoma (RCCC).

Methods: Between June 2012 and June 2015, RCCC tissues were obtained for the experimental group, and RCCC adjacent tumor-free kidney parenchyma tissues were obtained for the control group. VEGFA mRNA and protein expressions and phosphoinositide 3-kinase, serine/threoninespecific protein kinase (AKT), and phosphorylated-AKT protein expressions were detected. The chemically synthesized specific siRNA using RNA interference technology was used to inhibit VEGFA gene expression in human RCCC 786-O cells. The negative control (NC) group was transfected with $\mathrm{NC}$ sequence, and the blank group was transfected with no sequence. Flow cytometry, scratch test, and cell-penetrating experiment were used to detect cell proliferation, apoptosis, migration, and invasion of 786-O cells.

Results: Positive expression of VEGFA protein was $60.62 \%$ in RCCC tissue and $18.34 \%$ in adjacent tissue with statistically significant difference $(P<0.001)$. VEGFA protein and mRNA expressions were higher in RCCC tissue than those in adjacent tissue (both $P<0.01$ ). VEGF expression in RCCC tissue was associated with Fuhrman grading and American Joint Committee on Cancer staging (both $P<0.05$ ). After RCCC 786-O cells transfecting the VEGFA siRNA, the VEGFA mRNA and protein expressions and phosphoinositide 3-kinase and phosphorylated-AKT protein expressions were significantly decreased, cell proliferation was remarkably inhibited, cell apoptotic ratio was obviously increased, and migration distance and invasive cell number were markedly decreased compared to those in the NC group and the blank group (all $P<0.05$ ).

Conclusion: Inhibition of VEGFA inhibited proliferation, promoted apoptosis, and suppressed migration and invasion of RCCC 786-O cells. VEGF has a potential role in diagnosis and therapy of RCCC.

Keywords: 786-O, siRNA, transfection, cell biological behavior, PI3K/AKT, Fuhrman grading, AJCC staging, HK-2

\section{Introduction}

Accounting for $2 \%-3 \%$ of all adult malignant tumors, renal cell carcinoma (RCC) is the most lethal urologic cancer, and there are about 209,000 newly diagnosed RCC and 102,000 deaths caused by RCC per year worldwide. ${ }^{1}$ If detected at early stages, RCC can be cured surgically, but $\sim 30 \%$ of patients have metastatic RCC at diagnosis, while another $25 \%-30 \%$ of patients undergoing surgery will experience local or distant recurrence, mainly to the bone and lung, and once metastasis develops, the prognosis of RCC is poor with the 5 -year survival rate of $0 \%-10 \%$. ${ }^{1,2}$ There are several histological
Correspondence: Zheng-Yan Tang Department of Urology, Xiangya Hospital, Central South University, 87 Xiangya Road, Changsha 410008, People's Republic of China

Tel +86 I35073। 8268

Email xytzyan@163.com 
subtypes of RCC due to differences in incidence, biological behavior, and metastasis potential, and $\sim 75 \%$ of RCC patients were diagnosed with renal clear cell carcinoma (RCCC), whereas the papillary and chromophobe subtypes contribute $15 \%$ and $5 \%$, respectively. ${ }^{3,4}$ The incidence of RCCC in the People's Republic of China shows an increasing trend year by year, and RCCC has changeable biological behavior, complex etiology, and poor prognosis, and thus, it is of great importance to study the biological factors in the occurrence and development of RCCC. ${ }^{5}$

As an endothelial cell-specific protein, vascular endothelial growth factor (VEGF) acts as a mediator of angiogenesis and vasculogenesis by regulating the integrity and activity of endothelial cells, contributing to optimal vascular shape and function, and activating vascular endothelial cells and monocyte chemoattraction. ${ }^{6}$ VEGF A (VEGFA) is not only a potent angiogenic factor but also a survival factor for endothelial cells during tumor angiogenesis and can function in vascular permeability and antiapoptosis. ${ }^{7}$ Besides, acting as a potent tumor angiogenic factor, VEGFA can stimulate the growth of new blood vessels and thus provide tumors with oxygen and nutrients. ${ }^{8}$ It has been reported that tumor angiogenesis by activating the VEGF pathway results in improved blood supply and enriched source of nutrients for RCCC. ${ }^{9}$ Besides, anti-VEGF therapies for metastatic RCCC patients have induced response rates of $30 \%-40 \%$ and improved overall survival by targeting VEGF. ${ }^{10}$ Moreover, VEGF -2578C/A and $-1154 \mathrm{G} / \mathrm{A}$ polymorphisms were shown to be related with lower prognosis of RCC patients. ${ }^{11}$ However, there were few studies focused on the potential role of VEGFA on the biological behavior of RCCC cells except one study, which showed that miR-185 inhibits cell proliferation and induces cell apoptosis by targeting VEGFA directly in RCCC. ${ }^{12}$ Thus, our study was conducted to investigate VEGFA expression in RCCC tissues and cells and the effects of VEGFA on cell proliferation, apoptosis, migration, and invasion of RCCC cells. Thus, our study might provide a valuable marker for the development of RCCC, which is of great clinical significant.

\section{Materials and methods}

\section{Specimen collection and grouping}

Between June 2012 and June 2015, 35 RCCC patients (20 males and 15 females; age ranges $37-72$ years; average age 50.7 years) undergoing surgical resection at Xiangya Hospital, Central South University, were enrolled in our study, and their RCCC tissues were treated as experimental group. RCCC adjacent tumor-free kidney parenchyma tissues $(n=35)$ were treated as control group. Tumor histopathologic grading was based on the Fuhrman standard recommended by World Health Organization, ${ }^{13}$ and clinical staging was based on the American Joint Committee on Cancer (AJCC) criteria. ${ }^{14}$ Inclusion criteria of RCCC patients were as follows: patients were pathologically diagnosed as RCCC patients; patients received kidney radical resection or partial nephrectomy; and patients received no tumor-related chemotherapy, radiotherapy, and immunological therapy. Exclusion criteria were as follows: patients combined with malignant tumor in other organs or body parts; patients combined with severe renal parenchymal atrophy caused by severe hydronephrosis, hypertension, diabetes, and other diseases; and patients who also had nephritis. All specimens were obtained from fresh surgical resection specimen tissue avoiding cancer necrosis, and the specimens were directly frozen in liquid nitrogen and were stored at $-80^{\circ} \mathrm{C}$ for the next step. This study has been approved by the Xiangya Hospital, Central South University Ethics Committee, and the written consent of patients and their families was obtained. The ethical approval for this study conformed to the standards of the Declaration of Helsinki. ${ }^{15}$

\section{Immunohistochemistry detecting VEGFA protein expression}

Frozen tissues were sliced, shaken with phosphate-buffered saline (PBS) containing $0.1 \%$ bovine serum albumin, incubated with $3 \%$ hydrogen peroxide at room temperature for 10 minutes to block endogenous peroxidase activity, then rinsed with PBS, added to with 1:150 diluted VEGFA antibody (EMD Millipore, Billerica, MA, USA), and incubated overnight at $4^{\circ} \mathrm{C}$. The slices were again washed with PBS, horseradish peroxidase goat anti-mouse/rabbit IgG (secondary antibody) (EMD Millipore) was instantly added in each slice, and incubated at room temperature for 15 minutes. Then they were again washed with PBS, stained with $3,3^{\prime}$ diaminobenzidine, restained with hematoxylin, dehydrated with ethanol gradually, treated with dimethylbenzene to become transparent, and sealed with neutral gum. The negative control (NC) used PBS solution to replace primary antibody. The result was determined as follows: VEGFA protein was positively localized in the cytoplasm and presented brownish yellow granules. Ten high-power fields of each slice in the tumor area were randomly selected with 100 cells per field. The scores based on color intensity were as follows: 0 point for noncoloring, 1 point for light yellow, 2 points for yellow, and 3 points for brown yellow; and the scores based on positive cell percentage were as follows: 0 point for $<5 \%, 1$ point for $5 \%-25 \%, 2$ points for $26 \%-50 \%$, 3 points for $51 \%-75 \%$, and 4 points for $>75 \%$. Negative was 
determined when the multiple coloring intensity of the product of positive cell percentage was $0-3$ points, while positive was determined when the product was $\geq 4$ points. ${ }^{16}$

\section{Western blot detecting protein expressions}

Protein lysate (Nanjing KeyGEN BioTECH Development Co, Ltd, Nanjing, People's Republic of China) was added to the frozen tissue or cells in logarithmic growth phase to extract total proteins, and Bradford method (Nanjing KeyGEN BioTECH Development Co, Ltd) was used for protein quantification. Total proteins $(50 \mu \mathrm{g})$ were extracted using $12 \%$ sodium dodecyl sulfate polyacrylamide gel electrophoresis, were transferred to a polyvinylidene fluoride membrane (EMD Millipore, Billerica, MA, USA), and blocked with $5 \%$ skimmed milk powder at $37^{\circ} \mathrm{C}$ for 1 hour. VEGFA, phosphoinositide 3-kinase (PI3K), serine/threoninespecific protein kinase (AKT), and phosphorylated-AKT (p-AKT) monoclonal antibodies (1:1,000 dilution; Abcam, Cambridge, UK) were added to the blocked proteins, and the whole system was incubated overnight at $4{ }^{\circ} \mathrm{C}$. The membrane was washed with poly(butylene succinate-coterephthalate) for three times with 5 minutes for each time, and horseradish peroxidase-conjugated secondary antibody (1:4,000 dilution; Abcam) was added to incubate at room temperature for 1 hour. Electro-chemi luminescence liquid was formulated to conduct contact reaction with the positive side of polyvinylidene fluoride membrane for 3-5 minutes, and then electro-chemi luminescence liquid was drained. The protein bands were developed at darkroom, and ImageJ software was used to calculate the gray scale values of the bands. The relative expressions of proteins were calculated as the ratio of gray scale values of the targeted band to the reference glyceraldehyde-3-phosphate dehydrogenase (GAPDH) band. Each experiment was repeated three times, and the average values were calculated.

\section{Real-time quantitative polymerase chain reaction (RT-qPCR) detecting VEGFA mRNA expression}

MiRNeasy Mini Kit (Qiagen NV, Venlo, the Netherlands) was used to extract total RNA from frozen tissues or cells in the logarithmic growth phase. RNA sample $(5 \mu \mathrm{L})$ was diluted with 20 -fold RNA enzyme-free ultrapure water, and the absorbance of RNA sample was detected using an ultraviolet spectrophotometer at $260 \mathrm{~nm}$ and $280 \mathrm{~nm}$. RNA concentration and purity were detected, and the ratio of optical density (OD)260/OD280 between 1.7 and 2.1 indicated high purity of RNA, which can be used for the follow-up experiments. cDNA templates were synthesized by reverse transcription PCR amplification reaction using PCR instrument (PerkinElmer, Waltham, MA, USA), and RT-qPCR experiments were conducted by using the ABI7500 quantitative PCR instrument (Applied Biosystems Inc., Foster City, CA, USA). The reaction conditions were as follows: denaturation at $95^{\circ} \mathrm{C}$ for 3 minutes, 30 cycles of denaturation at $94^{\circ} \mathrm{C}$ for 30 seconds, annealing at $55^{\circ} \mathrm{C}$ for 30 seconds, and extension at $72^{\circ} \mathrm{C}$ for 30 seconds. The primers for VEGFA were as follows: 5'-TTGCTGCTCTACCTCCACCAT-3' (forward) and 5'-GGTGATGTTGGACTCCTCAGTG-3' (reverse); and the primers for GAPDH were as follows: 5'-TGAAGGTCGGAGTCAACGGA-3' (forward) and 5'-CCTGGAAGATGGTGATGGGAT-3' (reverse). GAPDH was used as an internal control, and each sample repeated measurements for three times. PCR results were analyzed with OpticonMonitor3 software (Bio-Rad Laboratories Inc., Hercules, CA, USA). The threshold was manually selected at the lowest point of each of the parallel rise of the logarithmic amplification curve to obtain $\mathrm{Ct}$ values of each reaction tube (threshold cycle), the data were analyzed using $2^{-\Delta \Delta \mathrm{Ct}}$ method $\left(2^{-\Delta \Delta \mathrm{Ct}}\right.$ represents the times ratio of target gene in the experimental group and the control group, and the formula was as follows: $\Delta \Delta \mathrm{Ct}=\Delta \mathrm{Ct}_{\text {experimental group }}-\Delta \mathrm{Ct} \mathrm{t}_{\text {control group }}$, wherein $\Delta \mathrm{Ct}=\mathrm{Ct}_{\text {target gene }}-$ $\left.\mathrm{Ct}_{\mathrm{GAPDH}}\right)$, and the experiment was repeated three times. ${ }^{17}$

\section{Cell culture}

Human RCCC 786-O and human renal tubular epithelial HK-2 cells were purchased from American Type Culture Collection and were cultured with RPMI 1640 medium (Hyclone, Logan, UT, USA) containing 10\% fetal bovine serum (FBS) (Thermo Fisher Scientific, Waltham, MA, USA), $100 \mathrm{U} / \mathrm{mL}$ penicillin, and $100 \mathrm{U} / \mathrm{mL}$ streptomycin (Hyclone) at $37^{\circ} \mathrm{C}$ in a $5 \%$ carbon dioxide $\left(\mathrm{CO}_{2}\right)$-saturated humidified incubator (Thermo Fisher Scientific) to conduct subculture. When the cells grew to a confluence of $80 \%, 0.25 \%$ trypsin (Beyotime Biotechnology Co, Ltd, Shanghai, People's Republic of China) was used for digestion, and the cells (cell density of $5 \times 10^{4}$ ) were subcultured and seeded in the culture flasks or plates. When the cells grew to a confluence of about $50 \%-70 \%$, the cells were used for the follow-up experiment.

\section{Cell transfection and VEGFA mRNA and protein expressions}

VEGFA siRNA and disorderly NC sequence were designed and synthesized by Shanghai Bioengineering Co, Ltd, (Shanghai, People's Republic of China) and the VEGFA siRNA sequences were as follows: 5'-CCGAAACCAUGAACUUUCUTT-3' (sense strand) and 5'-AGAAAGUUCAUGGUUUCGGTT-3' 
(antisense strand) (VEGFA siRNA-transfected group); and the disorderly $\mathrm{NC}$ sequences were as follows: 5'-UUCUCCGAACGUGUCACGUTT-3' (sense strand) and 5'-ACGUGACACGUUCGGAGAATT-3' (antisense strand) (NC group). Human RCCC at the logarithmic phase was seeded in 6-well plates, and when the cell confluence reached up to $60 \%-70 \%$, the medium was discarded; the siRNA was dissolved in Opti-MEM (Thermo Fisher Scientific), mixed with Lipofectamine RNAiMIX (Thermo Fisher Scientific), and added into each well of plates. The blank group only added the same amount of Opti-MEM low serum liquid nutrient medium. After culturing for 6 hours, old medium was removed out of wells, and fresh Dulbecco's Modified Eagle's Medium containing 10\% FBS was used for culture. Twenty-four hours after transfection, cells in each well were collected for the detection of VEGFA mRNA expression using the RT-qPCR method mentioned earlier; and 48 hours after transfection, cells in each well after lysis were collected for the detection of VEGFA, PI3K, AKT, and p-AKT protein expressions using the Western blot.

\section{Cell Counting Kit-8 (CCK-8) assay detecting cell proliferation}

After transfection, cells of each group in the logarithmic growth phase were seeded in 96-well plates, and cell numbers at specific time points were measured when the cells grew adherent to walls. The specific steps are as follows: the medium solution was discarded, $100 \mu \mathrm{L}$ of fresh medium containing $10 \mu \mathrm{L}$ CCK-8 reagent (Beyotime Biotechnology $\mathrm{Co}, \mathrm{Ltd})$ was added, well plates were placed in a $\mathrm{CO}_{2}$ incubator to conduct stand culture for 2 hours, and a microplate reader (Bio-Rad Laboratories Inc.) was used to measure OD at $450 \mathrm{~nm}$ wavelength. Each group had six repeat holes.

\section{Flow cytometry detecting cell apoptosis}

RCCC 786-O cells were collected 48 hours after transfection, and cell density was adjusted to $1 \times 10^{6} / \mathrm{mL}$. Cell suspension $(0.5 \mathrm{~mL})$ was placed in a centrifuge tube, $1.25 \mu \mathrm{L}$ of Annexin V-FITC (Nanjing KeyGEN BioTECH Development Co, Ltd) was added to the centrifuge tube, the whole system was carried out in a dark room at room temperature for 15 minutes and centrifuged at $1,000 \times g$ for 5 minutes, the supernatant was discarded, the cells were gently treated with $0.5 \mathrm{~mL}$ of cold-binding buffer to resuspend and $10 \mu \mathrm{L}$ of propidium iodide (PI), and flow cytometer (BD, Franklin Lakes, NJ, USA) was immediately used for detection analysis. The results are shown in the scatter plots: lower left quadrant (Q4, FITC-/PI-) showed healthy living cells, right lower quadrant (Q3, FITC+/PI-) showed early apoptotic cells, and right upper quadrant (Q2, FITC+/PI+) showed necrosis and late apoptosis cells. The apoptotic rate = early apoptotic cell percentage $(\mathrm{Q} 3)+$ late apoptotic cell percentage $(\mathrm{Q} 2)$.

\section{Scratch assay detecting cell migration}

Forty-eight hours after transfection, cells in each group were seeded in 6-well plates at a cell density of $5 \times 10^{5}$ cells/well. When the cells reached $80 \%$ confluence, $1 \mathrm{~mL}$ sterile pipette tip draw a vertical line from the middle of each hole from top to down, PBS was used to rinse cells, the medium was added into a $5 \% \mathrm{CO}_{2}$ incubator at $37^{\circ} \mathrm{C}$, and the cells were cultured for 24 hours, fixed with $75 \%$ alcohol at $4^{\circ} \mathrm{C}$ for 30 minutes, stained with hematoxylin-eosin, and sealed with neutral gum. The width variations in three crawling slides of each group were observed under a light microscope, and the average cell migration distances were measured using the Image-Pro Plus Analysis software.

\section{Transwell assay detecting cell invasion}

Matrigel gum $(40 \mu \mathrm{L})$ dissolved at $4^{\circ} \mathrm{C}$ was added into a precooled Transwell chamber and was incubated in a cell incubator for 1 hour to enable the gelation of the Matrigel. Cells in each group were taken after 48 hours of transfection, serum-free culture medium was used to adjust the cell concentration to $1 \times 10^{5} / 100 \mu \mathrm{L}, 100 \mu \mathrm{L}$ of cell suspension was added to the upper chamber of Transwell chamber (BD), $500 \mu \mathrm{L}$ of culture medium containing $10 \%$ FBS was added to the lower chamber, the cells were cultured in a $5 \%$ $\mathrm{CO}_{2}$ incubator at $37^{\circ} \mathrm{C}$ for 48 hours, the small chamber was removed, cotton swabs were used to wipe the cells from the upper chamber, $4 \%$ paraformaldehyde was used to fix the cells for 15 minutes, and the cells were washed with PBS once, stained with crystal violet for 10 minutes, and washed with PBS once. Five fields (up, down, left, right, and middle) were chosen to count the cells under a high-power microscope (Olympus Corporation, Tokyo, Japan), and the numbers of cells passing through the Matrigel membrane were calculated.

\section{Statistical analysis}

All data were analyzed using SPSS 18.0 statistical software (SPSS Inc., Chicago, IL, USA). Count data were expressed as percentage and tested using $\chi^{2}$-test, and measurement data were expressed as mean \pm standard deviation; the comparison between two groups was analyzed using two-sample $t$-test, and the comparison among multiple groups was analyzed using analysis of variance. Pearson correlation analysis 

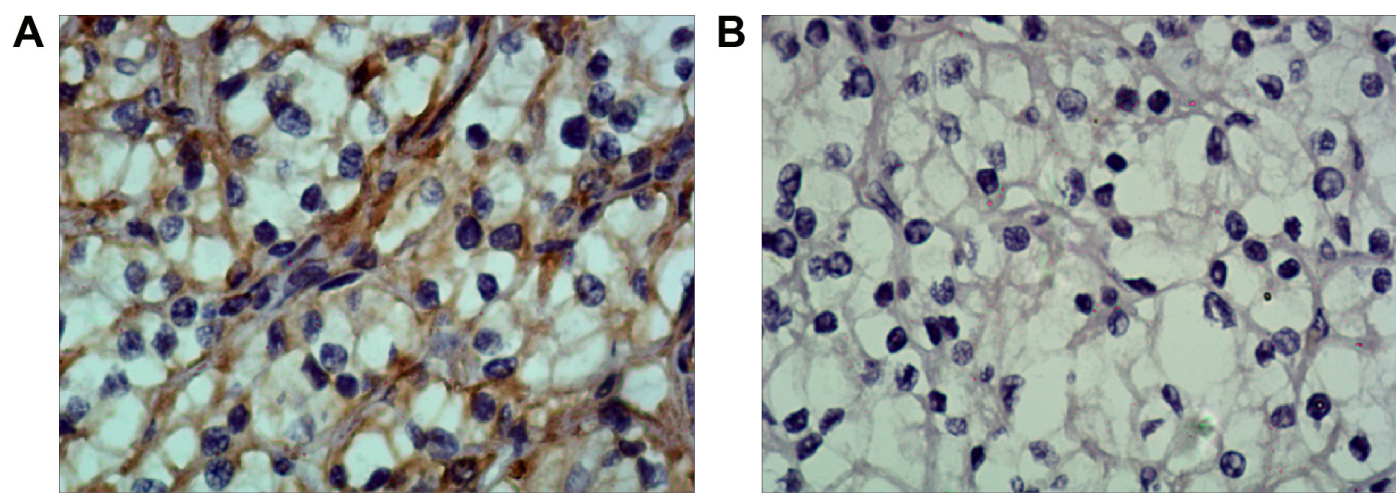

Figure I VEGFA protein expression.

Notes: VEGFA protein positively expressed in RCCC tissues $(\mathbf{A})$ and negatively expressed in adjacent tissues (B) detected by immunohistochemistry. Magnification $\times 400$. Abbreviations: RCCC, renal clear cell carcinoma; VEGFA, vascular endothelial growth factor A.

was performed for correlation analysis. $P<0.05$ indicates significant difference.

\section{Results}

\section{VEGFA expression in RCCC tissues} and adjacent tissues associated with clinicopathological features

Immunohistochemistry results showed that VEGFA stained darker in RCCC tissues than in the control group (Figure 1). In 35 cases of RCCC tissues, 21 cases had high expression of VEGFA, 14 cases had low expression of VEGFA, and the positive expression rate of VEGFA was $60.62 \%$ in RCCC tissues, while it was $18.34 \%$ in adjacent tissues, showing significant differences between the two groups $\left(\chi^{2}=13.57\right.$, $P<0.001$ ) (Table 1). As shown in Table 2, VEGFA protein expression was not associated with sex and age (both $P>0.05)$ but was associated with Fuhrman grading and AJCC staging (both $P<0.05$ ). The VEGFA protein expression in RCCC tissues was significantly higher than that in adjacent tumor-free kidney parenchyma tissues $(3.35 \pm 1.12$ vs $1.76 \pm 0.78 ; t=6.628, P<0.01$ ) (Figure $2 \mathrm{~A}$ ). Besides, the VEGFA mRNA expression in RCCC tissues was also obviously higher than that in adjacent tissues $(6.51 \pm 2.25 \mathrm{vs}$ $2.17 \pm 0.91 ; t=9.947, P<0.01$ ) (Figure 2B). VEGFA mRNA and protein expression levels were significantly positively correlated $(r=0.878, P<0.001)$.

Table I VEGFA expressions in RCCC tissues and adjacent tissues

\begin{tabular}{llllll}
\hline & Case & VEGFA & \multirow{2}{*}{$\chi^{2}$} & P-value \\
\cline { 3 - 5 } & & Positive (\%) & Negative (\%) & & \\
\hline RCCC tissues & 35 & $21(60.62 \%)$ & $14(39.39 \%)$ & 13.57 & $<0.00$ I \\
Adjacent tissues & 35 & $6(18.34 \%)$ & $29(81.67 \%)$ & & \\
\hline
\end{tabular}

Abbreviations: RCCC, renal clear cell carcinoma; VEGFA, vascular endothelial growth factor $\mathrm{A}$.

\section{VEGFA expression in RCCC 786-O and} renal tubular epithelial $\mathrm{HK}-2$ cells

The VEGFA protein expression was $2.45 \pm 0.59$ in RCCC $786-\mathrm{O}$ cells and $1.17 \pm 0.21$ in HK-2 cells with statistically significant difference $(t=3.534, P=0.024)$ (Figure $3 A)$. In addition, the VEGFA mRNA expression was $3.15 \pm 0.78$ in RCCC $786-\mathrm{O}$ cells and $1.41 \pm 0.27$ in HK-2 cells with statistically significant difference $(t=3.647, P=0.021)$ (Figure 3B).

\section{VEGFA expression and related proteins in PI3K/AKT pathway of transfected cells in each group}

After transfection of siRNA for 24 hours, VEGFA mRNA expression changes were detected by RT-qPCR. The results showed that the VEGFA mRNA expression $(0.93 \pm 0.15)$ was obviously decreased in the transfected group compared to that in the NC group and the blank group $(F=16.92$,

Table 2 Associations between VEGFA expressions and clinicopathological features of RCCC patients

\begin{tabular}{|c|c|c|c|c|c|}
\hline \multirow[t]{2}{*}{$\begin{array}{l}\text { Clinicopathological } \\
\text { features }\end{array}$} & \multirow[t]{2}{*}{ Case } & \multicolumn{2}{|c|}{$\begin{array}{l}\text { VEGFA } \\
\text { expressions }\end{array}$} & \multirow[t]{2}{*}{$\chi^{2}$} & \multirow[t]{2}{*}{$P$-value } \\
\hline & & Negative & Positive & & \\
\hline \multicolumn{6}{|l|}{ Sex } \\
\hline Male & 20 & 9 & 11 & 0.122 & 0.727 \\
\hline Female & 15 & 5 & 10 & & \\
\hline \multicolumn{6}{|l|}{ Age } \\
\hline$\leq 60$ & 22 & 8 & 14 & 0.046 & 0.830 \\
\hline$>60$ & 13 & 6 & 7 & & \\
\hline \multicolumn{6}{|l|}{ Fuhrman grading } \\
\hline I-II & 13 & 9 & 4 & 5.552 & 0.018 \\
\hline III-IV & 22 & 5 & 17 & & \\
\hline \multicolumn{6}{|l|}{ AJCC staging } \\
\hline I-II & 15 & 10 & 5 & 5.955 & 0.015 \\
\hline III-IV & 20 & 4 & 16 & & \\
\hline
\end{tabular}

Abbreviations: AJCC, American Joint Committee on Cancer; RCCC, renal clear cell carcinoma; VEGFA, vascular endothelial growth factor A. 
A

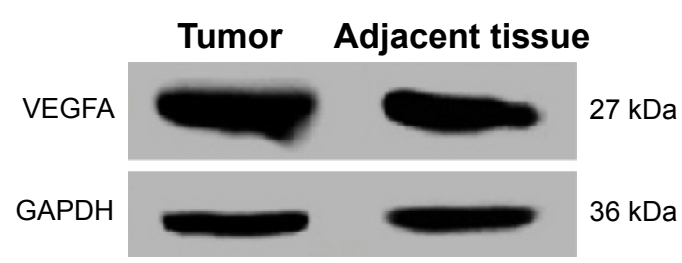

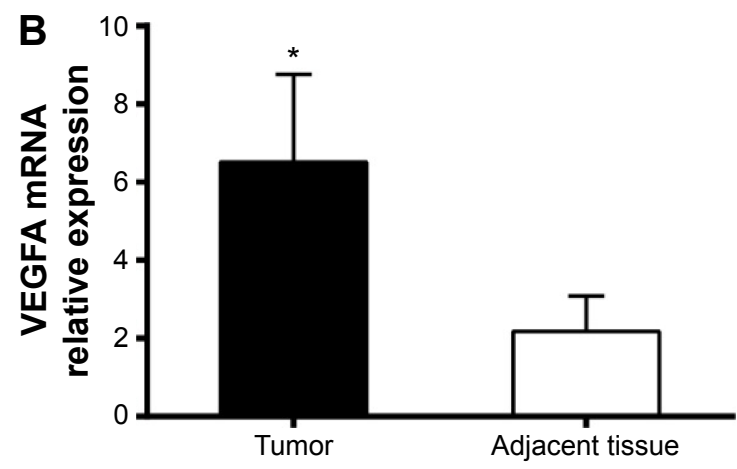

Figure 2 VEGFA expressions in RCCC tissues and adjacent tissues.

Notes: Western blot detecting protein expression $(\mathbf{A})$ and RT-qPCR detecting mRNA expression (B). *Compared to adjacent tissues, $P<0.05$.

Abbreviations: GAPDH, glyceraldehyde-3-phosphate dehydrogenase; RCCC, renal clear cell carcinoma; RT-qPCR, real-time quantitative polymerase chain reaction; VEGFA, vascular endothelial growth factor $A$.

$P=0.003)$. The VEGFA mRNA expression was $3.25 \pm 0.75$ in the NC group and 3.01 \pm 0.53 in the blank group, suggesting no significant difference between the two groups $(t=0.453$, $P=0.674$ ) (Figure 4A). After transfection of siRNA for 48 hours, VEGFA protein changes were detected by Western blot analysis. The VEGFA protein expression level decreased significantly in the transfected group $(0.89 \pm 0.05)$ compared to that in the NC group and the blank group ( $F=18.45$, $P=0.003$ ). There were no significant differences in VEGFA protein expression level between the $\mathrm{NC}$ group and the blank group $(2.76 \pm 0.53$ vs $2.43 \pm 0.45 ; t=0.822, P=0.457)$ (Figure $4 \mathrm{~B}$ and $\mathrm{C}$ ). PI3K/AKT pathway-related protein expression levels were shown (Figure $4 \mathrm{~B}$ and $\mathrm{C}$ ). There were no significant differences in the protein expressions between the NC group and the blank group (all $P>0.05$ ). $\mathrm{PI} 3 \mathrm{~K}$ and $\mathrm{p}$-AKT proteins were significantly decreased (all $P<0.05$ ), while AKT was slightly decreased in the VEGFA siRNA-transfected group compared to the NC group and the blank group, which indicated that VEGFA might regulate the biological behavior of the human RCCC through the PI3K/ AKT pathway (Figure 4B and C).

\section{Decreased cell proliferation after transfection of VEGFA siRNA}

CCK-8 was used to detect the cell proliferation activity of $786-\mathrm{O}$ cells in vitro, absorbance at $450 \mathrm{~nm}$ reflected the relative number of living cells, and the results are shown in Figure 5A. With the increase in time, the OD values of cells in each group increased. Immediately after transfection, the OD values of cells in the three groups were detected, and there was no statistically significant difference among the three groups $(P>0.05)$. Twenty-four hours after transfection, the OD value of 786-O cells was found significantly decreased in VEGFA siRNA-transfected group compared to that in the NC group and the blank group (both $P<0.05$ ). As can be seen from the proliferation curve (Figure $5 \mathrm{~B}$ ), the values in growth curve of VEGFA siRNA-transfected group were significantly lower than those in the NC group and the blank group 24 hours after transfection, indicating that the proliferation of 786-O cells was obviously inhibited, while the growth curve of NC group and blank group was overlapped, indicating that the proliferation abilities of 786-O cells in the two groups were not significantly different.
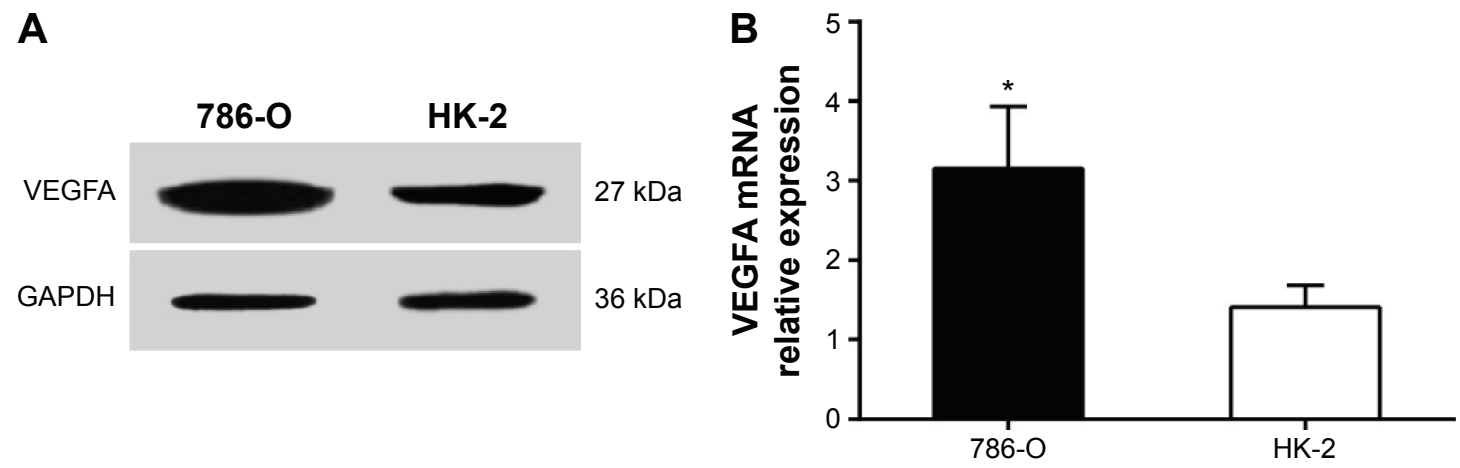

Figure 3 VEGFA expressions in human RCCC 786-O cells and renal tubular epithelial HK-2 cells.

Notes: Western blot detecting protein expression $(\mathbf{A})$ and RT-qPCR detecting mRNA expression (B). *Compared to HK-2 cells, $P<0.05$.

Abbreviations: GAPDH, glyceraldehyde-3-phosphate dehydrogenase; RCCC, renal clear cell carcinoma; RT-qPCR, real-time quantitative polymerase chain reaction; VEGFA, vascular endothelial growth factor $A$. 
A

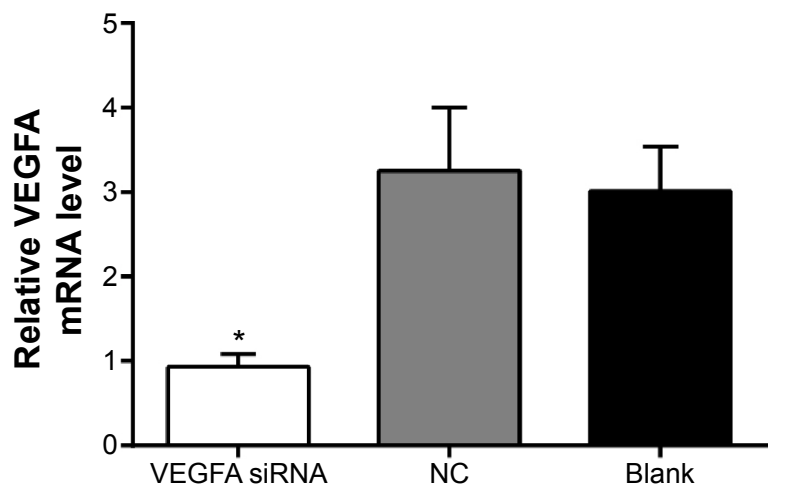

B

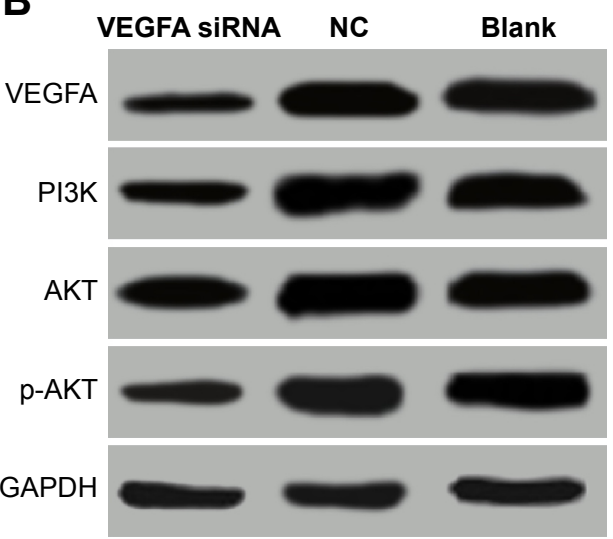

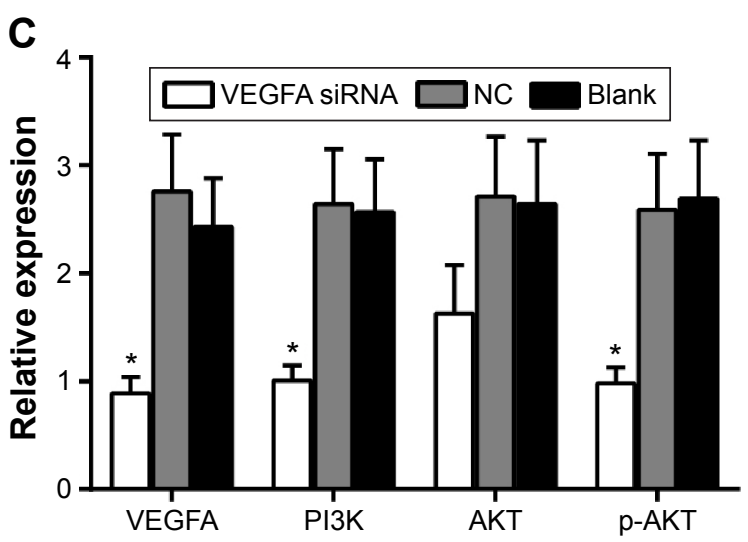

Figure 4 VEGFA expressions and PI3K/AKT pathway in human RCCC 786-O cells 24 hours after transfection in each group.

Notes: RT-qPCR detecting mRNA expression (A), Western blot detecting protein expressions (B), and histogram of protein expressions $(\mathbf{C})$. ${ }^{*}$ Compared to the NC group or the blank group, $P<0.05$.

Abbreviations: AKT, serine/threonine-specific protein kinase; GAPDH, glyceraldehyde-3-phosphate dehydrogenase; NC, negative control; PI3K, phosphoinositide 3-kinase; P-AKT, phosphorylated-AKT; RCCC, renal clear cell carcinoma; RT-qPCR, real-time quantitative polymerase chain reaction; VEGFA, vascular endothelial growth factor A.

\section{Increased cell apoptotic rate after transfection of VEGFA siRNA}

Flow cytometry was used to detect cell apoptotic rate 48 hours after transfection. The cell apoptotic rate was $19.23 \% \pm 1.21 \%$ in the VEGFA siRNA-transfected group, $12.96 \% \pm 1.01 \%$ in the $\mathrm{NC}$ group, and $11.78 \% \pm 0.92 \%$ in the blank group As shown in Figure 6, compared to the NC group and the blank group, the apoptotic rate in transfected group was significantly increased $(t=6.890, P=0.002 ; t=8.489, P=0.001$, respectively). On the contrary, compared to the $\mathrm{NC}$ group
A

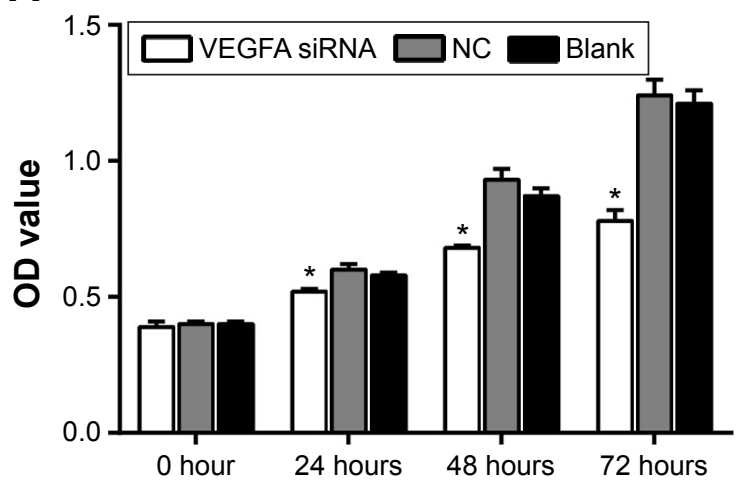

B

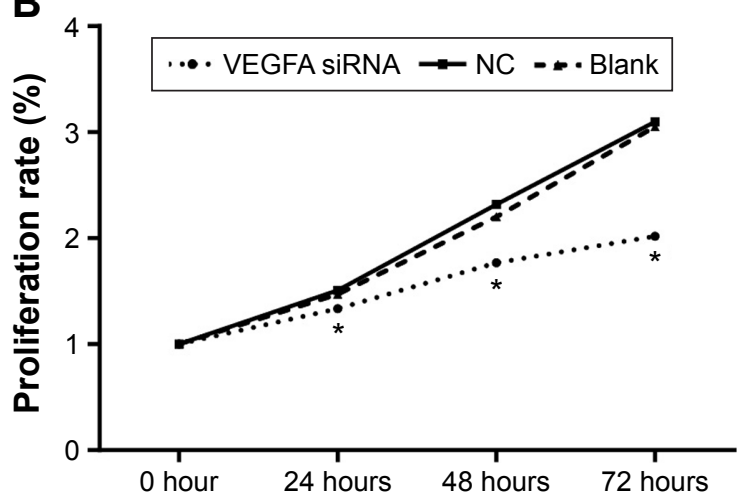

Figure 5 RCCC 786-O cell proliferation detected by CCK-8 assay.

Notes: Cell absorbance of $786-\mathrm{O}$ cells in each group $(\mathbf{A})$ and proliferation curve of $786-\mathrm{O}$ cells in each group $(\mathbf{B})$. $*$ Compared to the $\mathrm{NC}$ group or the blank group, $P<0.05$. Abbreviations: CCK-8, Cell Counting Kit-8; NC, negative control; OD, optical density; RCCC, renal clear cell carcinoma; VEGFA, vascular endothelial growth factor A. 

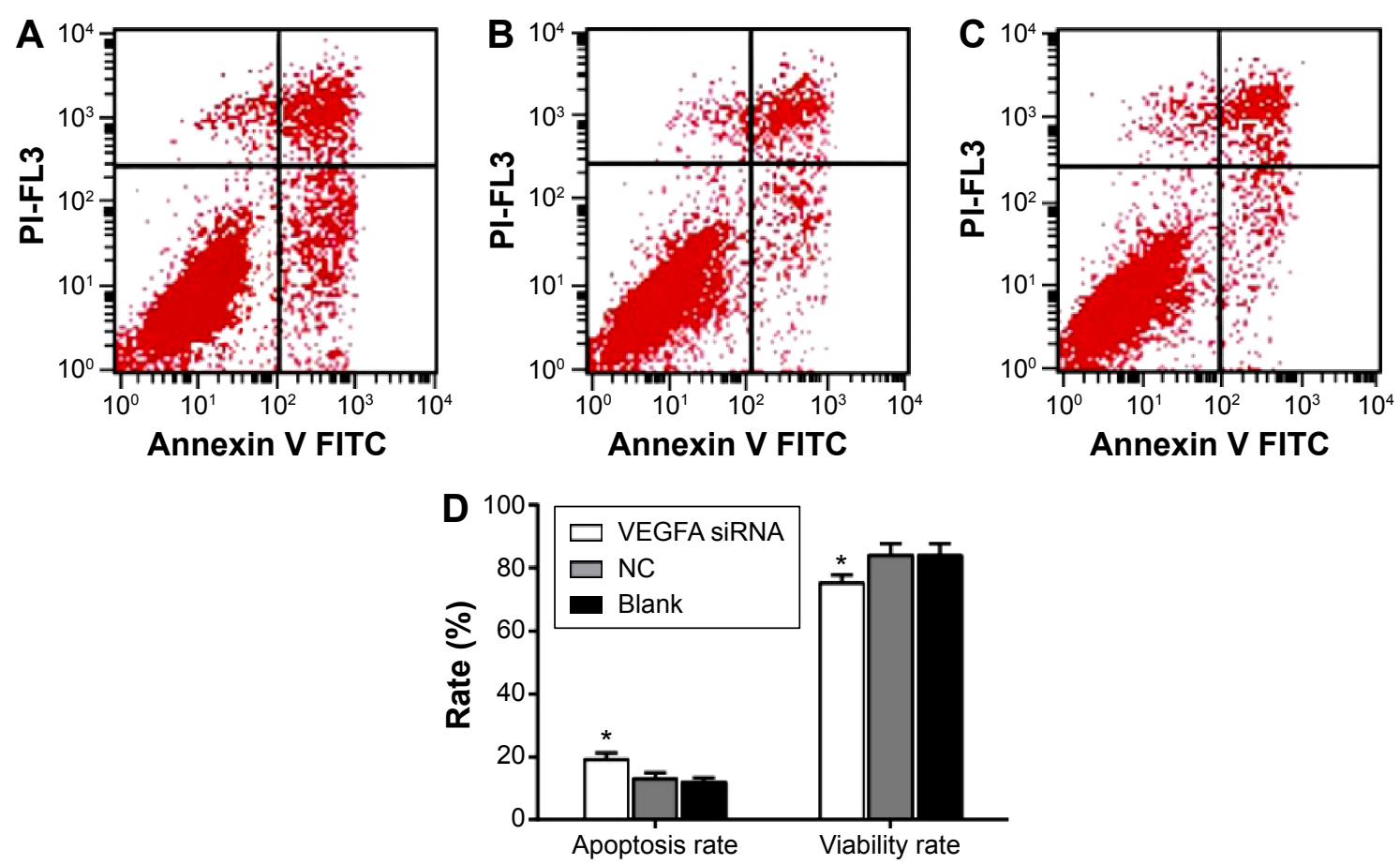

Figure 6 RCCC 786-O cell apoptosis in each group.

Notes: VEGFA siRNA-transfected group (A), NC group (B), blank group $(\mathbf{C})$, and apoptosis rate and viability rate in each group (D). *Compared to the NC group or the blank group, $P<0.05$.

Abbreviations: FL3, fluorescence 3; FITC, fluorescein isothiocyanate; NC, negative control; PI, propidium iodide; RCCC, renal clear cell carcinoma; VEGFA, vascular endothelial growth factor $\mathrm{A}$.

$(83.96 \% \pm 3.76 \%)$ and the blank group $(85.01 \% \pm 3.26 \%)$, live cell ratio in the VEGFA siRNA-transfected group $(75.23 \% \pm 2.58 \%)$ was significantly lower $(t=3.316, P=0.029$; $t=4.075, P=0.015$, respectively).

\section{Suppressed cell migration and invasion ability after transfection of VEGFA siRNA}

Scratch test results showed that the migration distance of the VEGFA siRNA-transfected group $(43.56 \pm 6.35 \mu \mathrm{m})$ was obviously decreased compared to that in the NC group $(73.36 \pm 7.32 \mu \mathrm{m})$ and the blank group $(72.12 \pm 7.13 \mu \mathrm{m})$ ( $t=5.326, P=0.006 ; t=5.181, P=0.007$, respectively) (Figure 7). Transwell results showed that invasive cell number was $25.60 \pm 2.77$ in the NC group and 25.15 \pm 2.82 in the blank group, showing no statistically significant difference $(t=0.197$, $P=0.853$ ), while the invasive cell number was $6.65 \pm 1.69$ in the VEGFA siRNA-transfected group and was obviously less than that in the $\mathrm{NC}$ group and the blank group $(t=10.12$, $P<0.001 ; t=9.747, P<0.001$, respectively), indicating that after transfection of VEGFA siRNA, migration and invasion capacity of the cells decreased significantly (Figure 8).

\section{Discussion}

In order to investigate VEGFA expression in RCCC tissues and cells and the effects of VEGFA on cell proliferation, apoptosis, migration, and invasion of RCCC cells, our study first studied the VEGFA expressions in RCCC tissues and adjacent healthy tissues. The results showed that VEGFA protein and mRNA expressions in RCCC tissues were significantly higher than those in adjacent tumor-free kidney parenchyma tissues. VEGFA is vital for normal glomerular function, and dysregulation of VEGFA might be associated with the development of glomerulopathies and disordered glycocalyx regulation. ${ }^{18}$ VEGFA levels and signaling play complex roles in maintaining glomerular endothelium viability but might cause endothelial cell pathology when the VEGFA levels are too high, for example, in diabetic nephropathy, VEGFA levels are transiently increased and lead to proteinuria. ${ }^{19}$ VEGFA and its soluble receptor 1 circulating levels are associated with inflammatory biomarkers, and VEGFA predicts all-cause mortality in incident dialysis patients..$^{20}$ Moreover, Yang et al showed that VEGFA polymorphisms were shown to be independent factors for the prognosis of RCC patients. ${ }^{11}$ Our study also indicated that VEGFA expressions were associated with RCCC patients. Since VEGFA expressions were an unfavorable prognostic factor for RCCC, VEGFA expressions could be used as a valuable biomarker for the prognosis of RCCC. Besides, our study also confirmed that VEGFA protein expression was associated with Fuhrman 

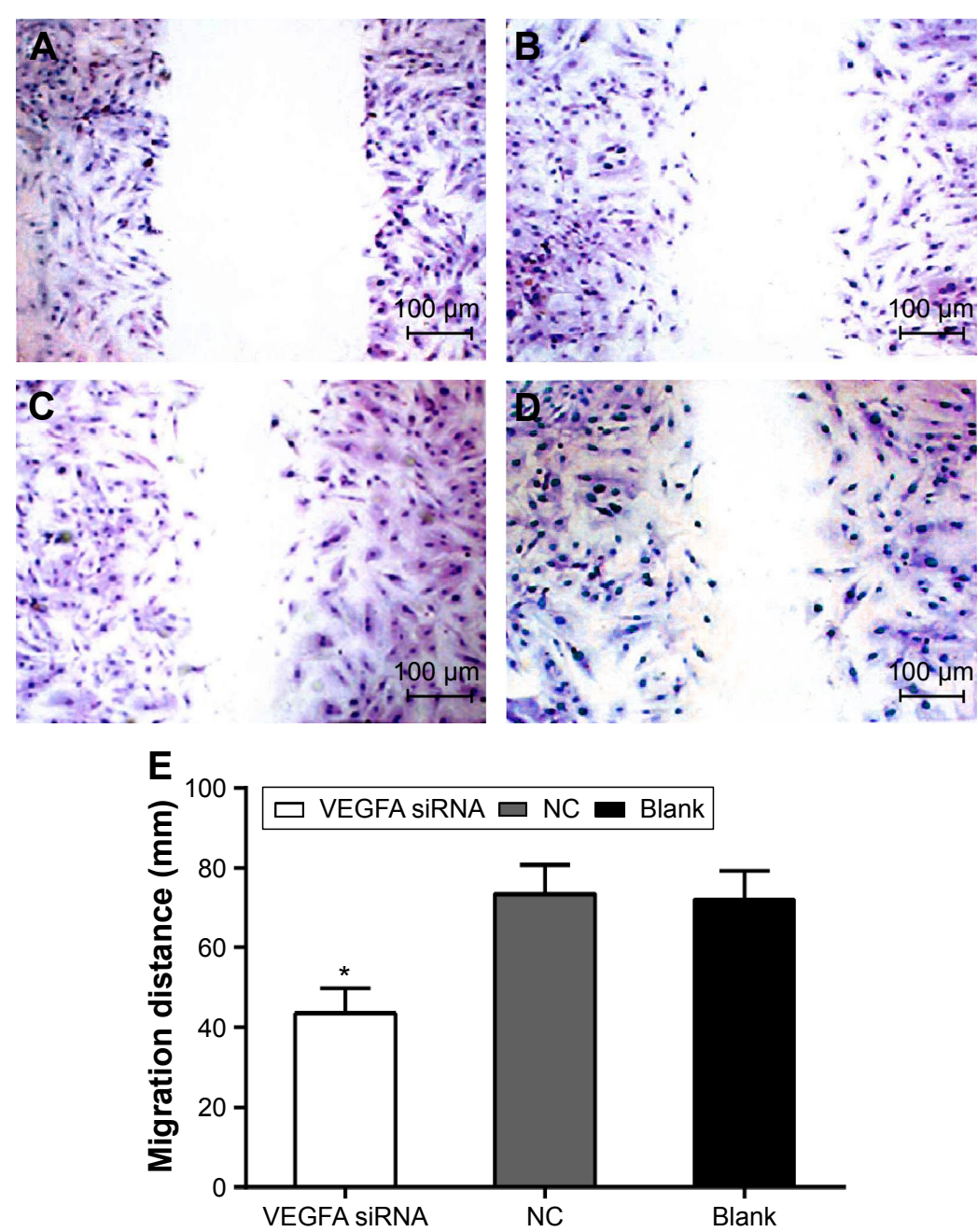

Figure 7 RCCC 786-O cell migration in each group.

Notes: Initial state of scratch (A), VEGFA siRNA-transfected group (B), NC group (C), blank group (D), and migration distance in each group (E). *Compared to the NC group or the blank group, $P<0.05$

Abbreviations: NC, negative control; RCCC, renal clear cell carcinoma; VEGFA, vascular endothelial growth factor A.
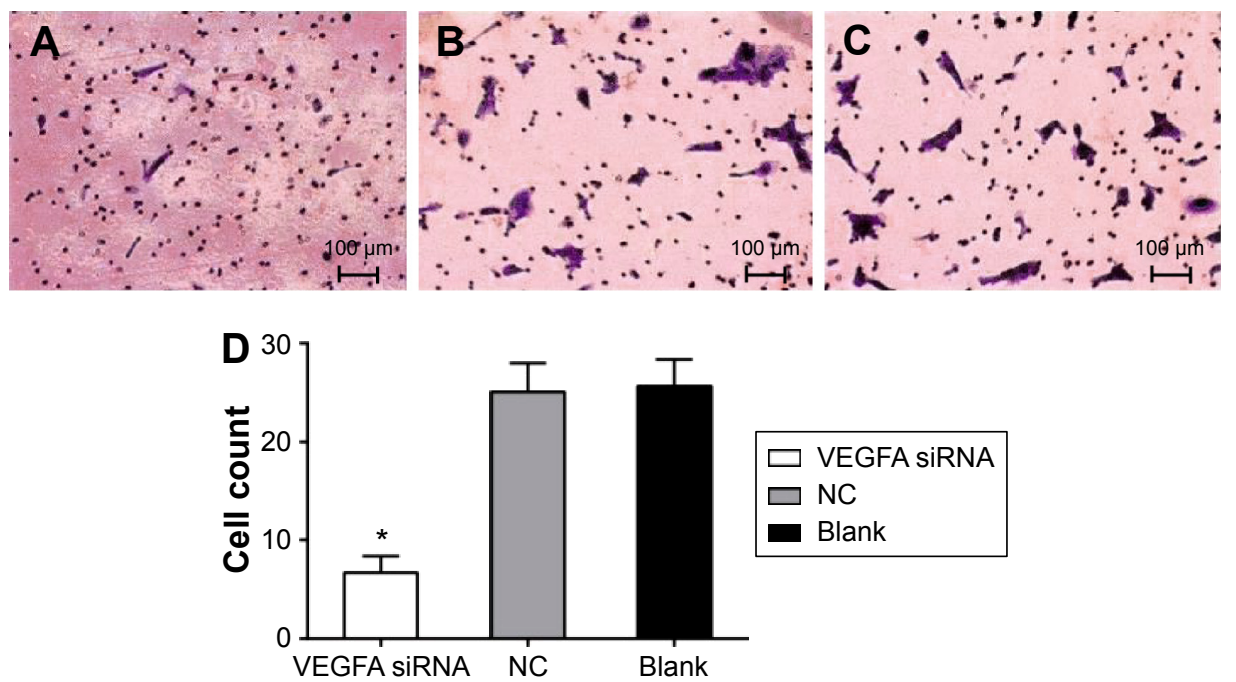

Figure 8 RCCC 786-O cell invasion in each group.

Notes: VEGFA siRNA-transfected group $(\mathbf{A}), \mathrm{NC}$ group $(\mathbf{B})$, blank group $(\mathbf{C})$, and invasive cell number in each group (D). ${ }^{*}$ Compared to the $\mathrm{NC}$ group or the blank group, $P<0.05$ Abbreviations: NC, negative control; RCCC, renal clear cell carcinoma; VEGFA, vascular endothelial growth factor A. 
grading and AJCC staging. Overexpression of VEGF may play important roles in the histological stages and clinical features of cutaneous squamous cell carcinoma. ${ }^{21}$ Liang et al showed that the expression of VEGF may be an important feature of advanced RCC. ${ }^{22}$

To further explore the association between VEGFA expressions and RCCC patients, we investigate the role of VEGFA expressions in human RCCC 786-O cells. Our results showed that after transfection of VEGFA siRNA in RCCC 786-O cells, the VEGFA mRNA and protein expressions and $\mathrm{PI} 3 \mathrm{~K}$ and $\mathrm{p}-\mathrm{AKT}$ proteins were significantly decreased, cell proliferation was remarkably inhibited, and cell apoptotic ratio obviously increased compared to those in the NC group and the blank group. It has been reported that VEGFA plays a critical role in the growth of primary tumors, is secreted by most solid tumors, and promotes endothelial cell proliferation, invasion, migration, and survival, as well as vessel permeability via the interaction of VEGFA and its receptor VEGF receptor-2 (VEGFR-2). ${ }^{23}$ In human colon cancer, high levels of VEGFA are associated with tumor progression and invasiveness, and thus, VEGFA expression has been found to be a strong prognostic indicator of colon cancer metastasis and survival. ${ }^{24}$ In neoplastic Barrett's cells, VEGF activation of VEGFR-2 initiates a phosphorylated phospholipase $\mathrm{C} \gamma 1$, protein kinase $\mathrm{C}$ (PKC), and extracellular signal-regulated kinases $1 / 2$ pathway that promotes proliferation and self-sustaining of neoplastic Barrett's cells. ${ }^{25}$ Furthermore, VEGFA is a positive regulator in cervical cancer angiogenesis, and the inhibition of VEGFA and its receptor signaling pathway can induce tumor cell apoptosis, suppress tumor angiogenesis, and inhibit tumor growth. ${ }^{26}$

AKT, also known as protein kinase B, played a pivotal role in cell proliferation, differentiation, and survival and is activated by a PI3K-dependent signaling pathway. ${ }^{27}$ The phosphorylation of AKT could activate downstream target genes involved in cell proliferation and cell cycle progression of tumor cells. ${ }^{28,29} \mathrm{PI} 3 \mathrm{~K} / \mathrm{AKT}$ signaling could activate noncanonical GLI1/2 in RCC and GLI1 and GLI2 are critical for RCC carcinogenesis. ${ }^{30}$ The proliferative actions of VEGF require activation of AKT signaling cascades, which are stimulated exclusively in actively proliferating neuronal progenitor cells. ${ }^{31}$ Thus, VEGF might promote the proliferation of RCCC by activating the PI3K/AKT signaling pathway, while inhibition of VEGF could inhibit the proliferation of RCCC.

Another important result of our study showed that migration distance and invasive cell number were markedly decreased in the VEGFA siRNA-transfected group compared to those in the NC group and the blank group. VEGFA functions in human hepatocellular carcinoma proliferation and migration are mediated by $\mathrm{P} 65, \mathrm{PKC} \alpha$, and/or p53 due to the fact that the knockdown of VEGF165 can decrease the expression of NF- $\mathrm{KB}$ p 65 and PKC $\alpha$ but can increase the expression of p53 signaling molecules. ${ }^{32}$ Glial cell line-derived neurotrophic factor enhances the migration of colon cancer cells by increasing VEGF-VEGFR interaction, mainly by regulating the p38, PI3K/AKT, and HIF1a signaling pathways. ${ }^{33}$ VEGFA induces endothelial and cancer cell migration by directly binding to integrin $\alpha 9 \beta 1$, which mediates accelerated cell adhesion and migration through interactions with diverse extracellular ligands. ${ }^{34}$ In addition, Vlajnic et al showed that $V E G F A$ gene locus (6p12) amplification could identify a small but highly aggressive subgroup of colorectal patients. ${ }^{35}$

\section{Conclusion}

In conclusion, our study showed that VEGFA protein and mRNA expressions were significantly higher in RCCC tissues and RCCC 786-O cells. Besides, the inhibition of VEGFA could inhibit cell proliferation, promote cell apoptosis, and suppress cell migration and invasion of RCCC 786-O cells. VEGFA might affect RCCC proliferation through the PI3K/AKT pathway. Our study could provide an important reference for the pathology of RCCC patients and provide a new direction for the treatment of RCCC patients. VEGF has a potential role in diagnosis and therapy of the RCCC.

\section{Acknowledgment}

We would like to give our sincere appreciation to the reviewers for their helpful comments on this article.

\section{Disclosure}

The authors report no conflicts of interest in this work.

\section{References}

1. Pan D, Xu L, Liu H, et al. Interleukin-11 receptor predicts post-operative clinical outcome in patients with early-stage clear-cell renal cell carcinoma. Jpn J Clin Oncol. 2015;45:202-209.

2. Pan D, Xu L, Liu H, et al. High expression of interleukin-11 is an independent indicator of poor prognosis in clear-cell renal cell carcinoma. Cancer Sci. 2015;106:592-597.

3. Girgis $\mathrm{H}$, Masui $\mathrm{O}$, White NM, et al. Lactate dehydrogenase A is a potential prognostic marker in clear cell renal cell carcinoma. Mol Cancer. 2014;13:101.

4. Gbormittah FO, Lee LY, Taylor K, Hancock WS, Iliopoulos O. Comparative studies of the proteome, glycoproteome, and N-glycome of clear cell renal cell carcinoma plasma before and after curative nephrectomy. J Proteome Res. 2014;13:4889-4900.

5. Gong BS, Feng Q. Netrin-1: the new tumor markers in renal clear cell carcinoma. Asian Pac J Trop Med. 2015;8:489-493. 
6. Almawi WY, Saldanha FL, Mahmood NA, Al-Zaman I, Sater MS, Mustafa FE. Relationship between VEGFA polymorphisms and serum VEGF protein levels and recurrent spontaneous miscarriage. Hum Reprod. 2013;28:2628-2635.

7. Su MT, Lin SH, Chen YC, Kuo PL. Gene-gene interactions and gene polymorphisms of VEGFA and EG-VEGF gene systems in recurrent pregnancy loss. J Assist Reprod Genet. 2014;31:699-705.

8. Geng L, Chaudhuri A, Talmon G, Wisecarver JL, Wang J. TGF-beta suppresses VEGFA-mediated angiogenesis in colon cancer metastasis PLoS One. 2013;8:e59918.

9. Joseph RW, Parasramka M, Eckel-Passow JE, et al. Inverse association between programmed death ligand 1 and genes in the VEGF pathway in primary clear cell renal cell carcinoma. Cancer Immunol Res. 2013;1: 378-385.

10. Wang Y, Choueiri TK, Lee JL, et al. Anti-VEGF therapy in mRCC: differences between Asian and non-Asian patients. Br J Cancer. 2014; 110:1433-1437.

11. Yang YQ, Chen J. Predictive role of vascular endothelial growth factor polymorphisms in the survival of renal cell carcinoma patients. Genet Mol Res. 2014;13:5011-5017.

12. Ma X, Shen D, Li H, et al. MicroRNA-185 inhibits cell proliferation and induces cell apoptosis by targeting VEGFA directly in von Hippel-Lindau-inactivated clear cell renal cell carcinoma. Urol Oncol. 2015;33:169.e1-e11.

13. Erdogan F, Demirel A, Polat O. Prognostic significance of morphologic parameters in renal cell carcinoma. Int J Clin Pract. 2004;58:333-336.

14. Edge SB, Compton CC. The American Joint Committee on Cancer: the 7th edition of the AJCC cancer staging manual and the future of TNM. Ann Surg Oncol. 2010;17:1471-1474.

15. PN M. World Medical Association publishes the revised Declaration of Helsinki. Natl Med J India. 2014;27:56.

16. Brown RS, Wahl RL. Overexpression of Glut-1 glucose transporter in human breast cancer. An immunohistochemical study. Cancer. 1993;72: 2979-2985.

17. Kim YK, Shin DH, Kim KB, et al. MUC5AC and MUC5B enhance the characterization of mucinous adenocarcinomas of the lung and predict poor prognosis. Histopathology. 2015;67:520-528.

18. Foster RR, Armstrong L, Baker S, et al. Glycosaminoglycan regulation by VEGFA and VEGFC of the glomerular microvascular endothelial cell glycocalyx in vitro. Am J Pathol. 2013;183:604-616.

19. Brosius FC, Coward RJ. Podocytes, signaling pathways, and vascular factors in diabetic kidney disease. Adv Chronic Kidney Dis. 2014;21: 304-310.

20. Yuan J, Guo Q, Qureshi AR, et al. Circulating vascular endothelial growth factor (VEGF) and its soluble receptor 1 (sVEGFR-1) are associated with inflammation and mortality in incident dialysis patients. Nephrol Dial Transplant. 2013;28:2356-2363.

21. An X, Xu G, Yang L, et al. Expression of hypoxia-inducible factor-1alpha, vascular endothelial growth factor and prolyl hydroxylase domain protein 2 in cutaneous squamous cell carcinoma and precursor lesions and their relationship with histological stages and clinical features. J Dermatol. 2014;41:76-83.
22. Liang YX, He HC, Han ZD, et al. CD147 and VEGF expression in advanced renal cell carcinoma and their prognostic value. Cancer Invest. 2009;27:788-793.

23. Qiu JF, Zhang ZQ, Wang Y, You J. Lentivirus-mediated RNAi knockdown of VEGFA in RKO colorectal cancer cells decreases tumor formation and growth in vitro and in vivo. Int J Clin Exp Pathol. 2012;5: 290-298.

24. Xing X, Gu X, Ma T, Ye H. Biglycan up-regulated vascular endothelial growth factor (VEGF) expression and promoted angiogenesis in colon cancer. Tumour Biol. 2015;36:1773-1780.

25. Zhang Q, Yu C, Peng S, et al. Autocrine VEGF signaling promotes proliferation of neoplastic Barrett's epithelial cells through a PLCdependent pathway. Gastroenterology. 2014;146(2):461-472.e6.

26. Qi L, Xing LN, Wei X, Song SG. Effects of VEGF suppression by small hairpin RNA interference combined with radiotherapy on the growth of cervical cancer. Genet Mol Res. 2014;13:5094-5106.

27. Wang HY, Yang SL, Liang HF, Li CH. HBx protein promotes oval cell proliferation by up-regulation of cyclin D1 via activation of the MEK/ ERK and PI3K/Akt pathways. Int J Mol Sci. 2014;15:3507-3518.

28. Jiang L, Wang C, Lei F, et al. miR-93 promotes cell proliferation in gliomas through activation of PI3K/Akt signaling pathway. Oncotarget. 2015;6:8286-8299.

29. Zhang C, Lan T, Hou J, et al. NOX4 promotes non-small cell lung cancer cell proliferation and metastasis through positive feedback regulation of PI3K/Akt signaling. Oncotarget. 2014;5:4392-4405.

30. Zhou J, Zhu G, Huang J, et al. Non-canonical GLI1/2 activation by PI3K AKT signaling in renal cell carcinoma: a novel potential therapeutic target. Cancer Lett. 2016;370(2):313-323.

31. Fournier NM, Lee B, Banasr M, Elsayed M, Duman RS. Vascular endothelial growth factor regulates adult hippocampal cell proliferation through MEK/ERK- and PI3K/Akt-dependent signaling. Neuropharmacology. 2012;63:642-652.

32. Zhang L, Wang JN, Tang JM, et al. VEGF is essential for the growth and migration of human hepatocellular carcinoma cells. Mol Biol Rep. 2012;39:5085-5093.

33. Huang SM, Chen TS, Chiu CM, et al. GDNF increases cell motility in human colon cancer through VEGF-VEGFR1 interaction. Endocr Relat Cancer. 2014;21:73-84.

34. Oommen S, Gupta SK, Vlahakis NE. Vascular endothelial growth factor A (VEGF-A) induces endothelial and cancer cell migration through direct binding to integrin $\alpha 9 \beta 1$ : identification of a specific $\alpha 9 \beta 1$ binding site. J Biol Chem. 2011;286:1083-1092.

35. Vlajnic T, Andreozzi MC, Schneider S, et al. VEGFA gene locus (6p12) amplification identifies a small but highly aggressive subgroup of colorectal cancer [corrected] patients. Mod Pathol. 2011;24:1404-1412.
OncoTargets and Therapy

\section{Publish your work in this journal}

OncoTargets and Therapy is an international, peer-reviewed, open access journal focusing on the pathological basis of all cancers, potential targets for therapy and treatment protocols employed to improve the management of cancer patients. The journal also focuses on the impact of management programs and new therapeutic agents and protocols on

\section{Dovepress}

patient perspectives such as quality of life, adherence and satisfaction. The manuscript management system is completely online and includes a very quick and fair peer-review system, which is all easy to use. Visit http://www.dovepress.com/testimonials.php to read real quotes from published authors. 